\title{
EFECTOS DE LA APLICACIÓN DE PERÓXIDO DE HIDRÓGENO AL 35\% Y CEPILLADO SOBRE EL ESMALTE DENTAL
}

\author{
${ }^{1}$ John Alexis Domínguez, ${ }^{2}$ María Alejandra Paredes Marín, ${ }^{2}$ Karol Andrea Gómez Ballesteros, ${ }^{2}$ Gustavo Adolfo Martínez Alvarado, \\ ${ }^{3}$ Gustavo Hannover Alegría Acevedo, ${ }^{4}$ Osnara María Mongruel Gomes \\ ${ }^{1}$ Odontólogo U. Santiago de Cali, Magíster en Odontología U. Estadual de Ponta Grossa, Candidato a Doctorando en Odontología U. Estadual de Ponta Grossa, Brasil. ${ }^{2}$ Estudiante X \\ Semestre F. Odontología U. Santiago de Cali, Colombia. ${ }^{3}$ Director Programa de Odontología, MSc en Odontología U. Santiago de Cali, Colombia. ${ }^{4}$ Doctorado en Odontología Restaura- \\ dora, Directora Pos Graduación U. Estadual de Ponta Grossa, Brasil.
}

Autor responsable de correspondencia: John Alexis Domínguez

Correo electrónico: johnalexis.dominguez@gmail.com

\section{RESUMEN}

Objetivo: evaluar los efectos sobre la dureza superficial y la rugosidad de un gel clareador de peróxido de hidrógeno 35\% con posterior cepillado.

Materiales y métodos: se realizó un estudio experimental In vitro, fueron realizados cuarenta bloques de esmalte de $3 \mathrm{~mm}^{2}$ de terceros molares en la porción vestibular, fueron lijados con lijas de granulación 600 - 1500, para ser divididos en las dos metodologías: microdureza Vickers $(n=20)$ y microscopia de fuerza atómica $(n=20)$. Estos fueron divididos en dos grupos, GC y GCC. En el grupo GC ( $n=10)$ se realizó aclaramiento con peróxido de hidrógeno al 35\% mientras que en el GCC, el aclaramiento se hizó con peróxido de hidrógeno al 35\% y posterior a éste se realizó cepillado con una crema dental que contenía 1450 ppm de flúor. Se realizó un test de ANOVA, un post test $t$ de Student con un nivel de significancia $\alpha \leq 0,05$, para conocer las diferencias entre los grupos.

Resultados: se encontró una diferencia estadísticamente significativa sobre el numero de microdureza vickers (NDV) en el grupo GCC (antes: 369,4 \pm 19,4 NDV, después: 392,4 \pm 14,2 NDV) pero no se observó ninguna diferencia estadísticamente significativa sobre la rugosidad ( $\mathrm{Ra})$, en ninguno de los dos grupos.

Conclusión: el cepillado con crema dental con 1450 ppm de fluor, después del proceso de aclaramiento dental con peróxido de hidrógeno 35\% mantiene la estabilidad de la dureza superficial del esmalte. [Domínguez JA, Paredes MA, Gómez KA, Martínez GA, Alegría GH, Gomes OMM. Efectos sobre esmalte de aplicación con peróxido de hidrógeno al 35\% y cepillado. Ustasalud 2013; 12: 41 - 46]

Palabras clave: Blanqueamiento de dientes, Cepillado dental, Pastas de Dientes, Esmalte

\section{EFFECTS ON DENTAL ENAMEL AFTER WHITENING WITH 35\% HYDROGEN PEROXIDE AND TOOTH BRUSHING}

\begin{abstract}
Objective: to evaluate the effects on surface hardness and roughness of the whitening gel 35\% hydrogen peroxide with brushed back. Methods: an experimental In vitro study was done, 40 blocks of $3 \mathrm{~mm}^{2}$ enamel of third molar teeth of the vestibular portion was smoothed with sandpaper blocks granulation from 600 to 1500, to be divided into the two methodologies, microhardness Vickers ( $\mathrm{n}=$ $20)$ and Atomic force Microscopy $(n=20)$, and this in two groups, GC and GCC. In the GC group the whitening was done with hydrogen peroxide $35 \%(\mathrm{n}=10$ ), in the GCC, it was performed with $35 \%$ hydrogen peroxide and brushing with toothpaste containing 1450 ppm of fluoride. ANOVA test and a post Student t test with a level of significance $\alpha=0.05$, was conducted to know the differences between the groups.

Results: a statistically significant difference on the number of Vickers microhardness (NDV) in the GCC group (before: $369.4 \pm 19.4$ NDV, after: $392.4 \pm 14.2 \mathrm{NDV}$ ) was found, but no statistically significant difference was observed for the surface roughness (Ra), between this two groups.

Conclusion: brushing with toothpaste containing $1450 \mathrm{ppm}$ fluoride after dental bleaching process with $35 \%$ hydrogen peroxide maintained the stability of the surface hardness of the enamel.
\end{abstract}

Key Words: Tooth Bleaching, ToothBrushing, Toothpastes, Enamel. 


\section{INTRODUCCIÓN}

El crecimiento de procedimientos de aclaramientos dentales viene siendo cada vez mayor, ${ }^{1}$ tanto por la facilidad para el clínico como por las necesitadas solicitadas por los pacientes. La anterior observación se demuestra con el estudio de Tin-Oo y colaboradores (2011), en el que entrevistaron a 235 pacientes sobre la satisfacción con su apariencia general; entre los resultados, $132(56,2 \%)$ pacientes, no estaban contentos con el color de sus dientes. ${ }^{2}$

La técnica de aclaramiento dental en el consultorio es una de las posibilidades para cambiar esta insatisfacción de los pacientes, requiere de altas concentraciones de Peróxido de Hidrógeno o Peróxido de Carbamida junto con una observación directa del clínico., ${ }^{3,4}$ Existe controversia sobre los efectos adversos de estos peróxidos en las estructuras del diente, uno de ellos se relaciona con la dureza del esmalte. Attin y colaboradores (2009) revisaron 55 estudios en los que utilizaron peróxido de hidrógeno en concentraciones mayores a $10 \%$, encontraron que el $50 \%$ obtuvó disminución de la dureza superficial y el otro $50 \%$ no, en contraste con los que utilizaron peróxido de carbamida mayor a $10 \%$, en $60 \%$ disminuyó esta dureza y en el $40 \%$ no. ${ }^{5}$

Otras de la evaluaciones realizadas son las alteraciones morfológicas como rugosidad y porosidad; estudios con microscopia electrónica de barrido han reportado la no alteración estructural con altas concentraciones de peróxido de hidrógeno..$^{6-9}$ Sin embargo, otros estudios han encontrado significativos efectos deletéreos sobre la estructura del esmalte como depresiones superficiales, diversos grados de aumento de la porosidad y aumento de la rugosidad. ${ }^{10-13} \mathrm{Un}$ estudio realizado por Domínguez y colaboradores, en 2012, evaluó la rugosidad de dos peróxidos de hidrógeno a altas concentraciones (35\% y 38\%) y se obtuvó entre sus resultados un aumento significativo de $\mathrm{Rz}$, con el peróxido de hidrógeno al 35\% y ningún efecto con el peróxido de hidrógeno al 38\%. ${ }^{14}$
Se ha investigado el uso del flúor al utilizarlo como aplicación tópica o incorporado a los productos de aclaramiento, para el mantenimiento de los valores de dureza superficial sin modificación estructural. ${ }^{14}$ Otros procedimientos diarios realizados por los pacientes en su higiene bucal como el cepillado dental con cremas dentales pueden ayudar a mantener estos valores como también pueden tener efectos adversos sobre la estructura dental. ${ }^{15}$ Por eso, el objetivo de este estudio fue evaluar los efectos sobre la dureza superficial y la rugosidad del esmalte de un gel clareador de peróxido de hidrógeno al 35\% con un cepillado posterior.

\section{MATERIALES Y MÉTODOS}

Se realizó un estudio experimental In vitro, fueron seleccionados veinte dientes terceros molares, libres de caries dental, previa aprobación de Comité de Ética de la Universidad Santiago de Cali por medio de Acta $\mathrm{N}^{\circ}$ 03-011B.

Los dientes se obtuvieron en las Clínicas de Cirugía de la Universidad Santiago de Cali y cada uno de los pacientes firmó un consentimiento informado para autorizar la donación de éste a la investigación. Los dientes fueron almacenados en timol al 0,1\% por un mes, tiempo que demoró la recolección de la muestra.

\section{Preparación de bloques de esmalte}

Los dientes fueron cortados $1 \mathrm{~mm}$ debajo de la unión amelocementaria (Figura $1 \mathrm{~A}, \mathrm{~B}$ ) con un disco de diamante, se realizó también un corte con orientación del eje longitudinal del diente de mesial a distal para crear dos caras (Vestibular y Palatina) (Figura $1 \mathrm{C}, \mathrm{D})$, es importante señalar que para este estudio sólo fueron utilizadas las caras vestibulares. Se realizaron dos bloques de $3 \mathrm{~mm} \times 3 \mathrm{~mm}$ (Figura $1 \mathrm{E}$, F), ${ }^{14}$ y se obtuvieron un total de cuarenta muestras que fueron alisadas con lijas de granulación 600 , 100, 1200 y 1500 (3M, Sumare, SP, Brasil) para realizar una distribución aleatoria simple en las metodologías utilizadas (Figura 2).

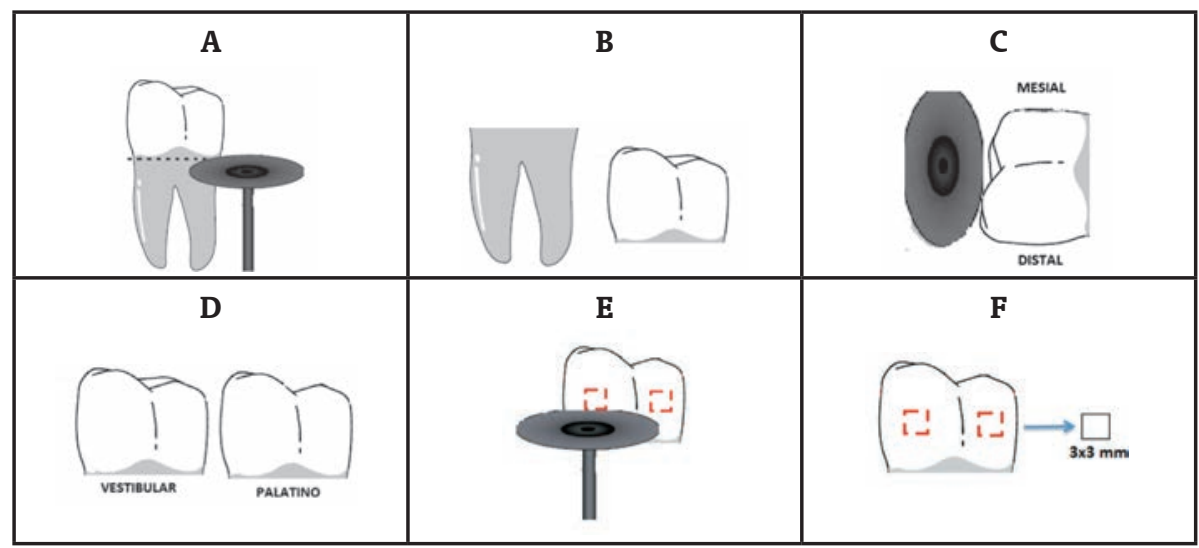

Figura 1. A: corte $1 \mathrm{~mm}$ debajo de la unión amelocementaria. B: corte raíz. C: corte eje longitudinal del diente. D: separación vestibular y palatino. E: corte de los bloques de esmalte. F: bloques de esmalte de $3 \mathrm{~mm}^{2}$. 


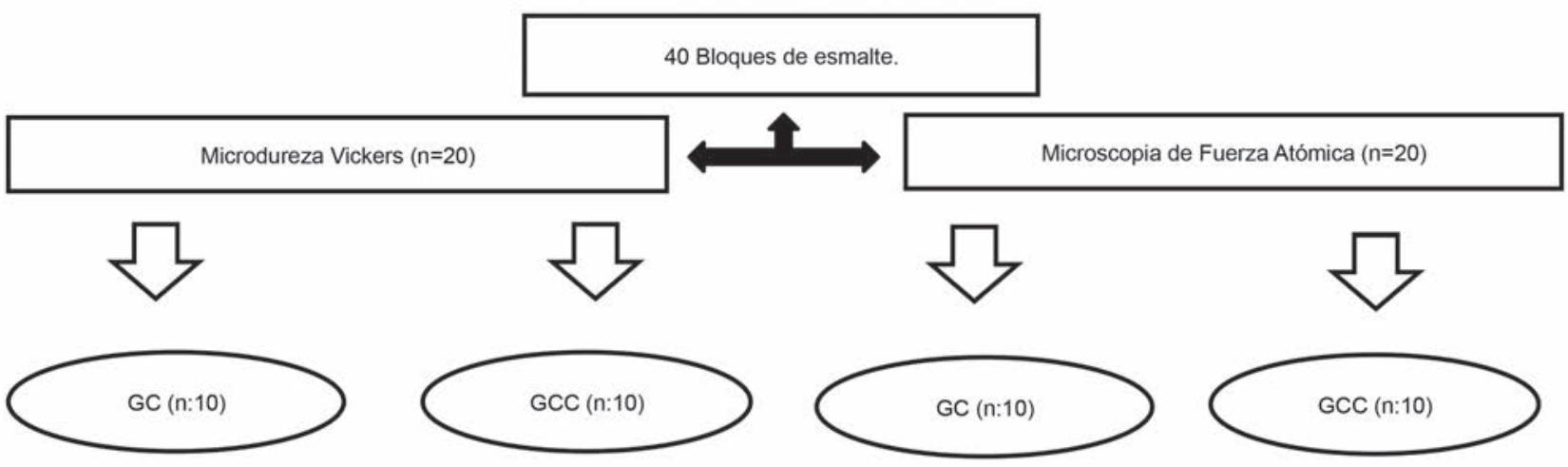

Figura 2. Distribución de los bloques de esmalte por metodología y grupos experimentales

Posterior a la distribución aleatoria se procedió a realizar los siguientes procedimientos experimentales:

Grupo C (GC): se realizó aclaramiento con peróxido de hidrógeno al 35\% (Whiteness HP Blue, Dentscare Ltda, Industria Brasileira, FGM) según las instrucciones del fabricante (Tabla 1).
Grupo CC (GCC): se le realizó aclaramiento con peróxido de hidrógeno al 35\% (Whiteness HP Blue Dentscare Ltda, Industria Brasileira, FGM) según las instrucciones del fabricante, y después se realizó el cepillado, con cepillo dental eléctrico (Colgate Motion, ColgatePalmolive Company, Cali - Colombia) y crema dental (Colgate máxima protección anti caries Colgate-Palmolive Company, Cali - Colombia) (Tabla 1).

Tabla 1. Materiales utiizados

\begin{tabular}{cccc}
\hline Material & Fabricante & Composición & Lote \\
\hline Whiteness HP Blue & FGM: Joinville-Santa Catarina \\
Brasil & $\begin{array}{c}\text { Peróxido de Hidrogeno 35\%, } \\
\text { espesante, pigmento inerte violeta, } \\
\text { agentes neutralizantes, gluconato de } \\
\text { calcio, glicol y agua ionizada }\end{array}$ \\
Colgate máxima & Colgate-Palmolive Company, \\
protección anti caries & Cali - Colombia & $\begin{array}{c}\text { Monofluorfosfato de Sodio 0,76\% y } \\
\text { Fluoruro de Sodio 0,1\% equivalente } \\
\text { a 1450 ppm, Fosfato de Calcio }\end{array}$ & Dihidratado. \\
\hline
\end{tabular}

\section{Protocolo experimental de cepillado In vitro}

Las muestras fueron fijadas en un cilindro acrílico de $8 \mathrm{~mm}$ de diámetro para evitar movimiento durante el proceso de cepillado, el operador presiona con el cepillo la balanza digital (Shimadzu Modelo AY, Kyoto Japon) a $250 \mathrm{gm}$ tres veces y en la cuarta realiza el cepillado con la crema dental (Colgate máxima protección anti caries ColgatePalmolive Company, Cali - Colombia) la cual contenia $1450 \mathrm{ppm}$ de flúor, fue mezclada con $1 \%$ de agua para obtener una viscosidad adecuada y estandarizada y obtener una buena dispersión de la suspensión con propiedades reológicas parecidas a las de la saliva. ${ }^{16-17}$
Las muestras de esmalte fueron cepilladas por diez minutos con cepillo dental eléctrico (Colgate Motion, Colgate-Palmolive Company, Cali - Colombia), a 120 ciclos por minuto con una carga aplicada de $250 \mathrm{gm}(2,5$ a $3 \mathrm{~N})$, después fueron lavadas con abundante agua ionizada. ${ }^{18}$

\section{Microdureza}

Se realizo la evaluación de la microdureza Vicker con microdurometro (Leica VMHT MOT, Leica Microsystem Alemania) sobre la superficie de esmalte después de los procedimientos experimentales al ubicar la muestra a 50X, con una carga de 50 gramos, con un tiempo de car- 
ga de diez segundos. Se realizaron seis indentaciones en cada bloque con distancias de 100 micras entre ellas. ${ }^{19}$

\section{Microscopia de fuerza atómica}

Los bloques fueron analizados por medio del Microscopio de Fuerza Atómica (AFM) (SPM-9600, Shimadzu, Kyoto, Japon), previa colocación de la muestra a $2 \mathrm{~mm}$ de altura y $3 \mathrm{~mm}$ de diámetro con cianocrilato (Pegamil, Anaerobicos ITW company, Sao Jose, Santa Catarina, Brasil). El análisis se realizó a modo contacto con una fuerza constante y la punta utilizada fue un cantiliver estándar de nitrato de silicio con una constante elástica de $0,01-1,0 \mathrm{~N} / \mathrm{m}$, en una área de $30 \mu \mathrm{m}$, fueron realizadas tres barreduras por bloque. ${ }^{14}$

\section{Análisis estadístico}

Para el análisis estadístico se utilizó el software BioEstat 5.0, se realizó el test de normalidad
D’Agostino y después fue realizado ANOVA con post test t de Student con nivel de significancia $\alpha \leq 0,05$.

\section{RESULTADOS}

\section{Microdureza}

Los valores del promedio y sus desviaciones estándar ( $\pm \mathrm{DE})$, se observan en la Tabla 2. Se presentó una diferencia significativa $(\mathrm{p}<0.001)$ entre los valores de antes y después de ambos grupos, incrementando los valores de microdureza Vicker en el grupo GCC y disminuyendo en el GC en un $6 \%$ cada uno.

\section{Microscopia de Fuerza Atómica}

Los valores del promedio y sus desviaciones estándar( $\pm \mathrm{DE})$ se muestran en la Tabla 3. Estos se refieren a la rugosidad (Ra) antes y después del aclaramiento. No se encontró una diferencia estadísticamente significativa $(p=0,56)$ en ninguno de los grupos (Figuras 3 y 4 ).

Tabla 2. Datos de microdureza Vicker antes y después del aclaramiento

\begin{tabular}{ccc}
\hline Grupo & $\begin{array}{c}\text { Antes } \\
\mathbf{x} \pm \mathrm{DE}\end{array}$ & $\begin{array}{c}\text { Después } \\
\mathbf{x} \pm \mathrm{DE}\end{array}$ \\
\hline GC & $373,5 \pm 10,4 \mathrm{~A}$ & $354,5 \pm 15,1 \mathrm{~B}$ \\
GCC & $369,4 \pm 19,4 \mathrm{~A}$ & $392,4 \pm 14,2 \mathrm{~B}$ \\
\hline
\end{tabular}

Letras diferentes denotan diferencia significativa.

Tabla 3. Datos de rugosidad presentados en porcentajes antes y despues del aclaramiento

\begin{tabular}{ccc}
\hline Grupo & $\begin{array}{c}\text { Antes } \\
\mathbf{x} \pm \mathrm{DE}\end{array}$ & $\begin{array}{c}\text { Después } \\
\mathbf{x} \pm \mathrm{DE}\end{array}$ \\
\hline GC & $11,3 \pm 5,6 \mathrm{~A}$ & $12,6 \pm 5,6 \mathrm{~A}$ \\
GCC & $10,3 \pm 5,7 \mathrm{~A}$ & $11,6 \pm 6,3 \mathrm{~A}$ \\
\hline
\end{tabular}

Letras diferentes denotan diferencia significativa.

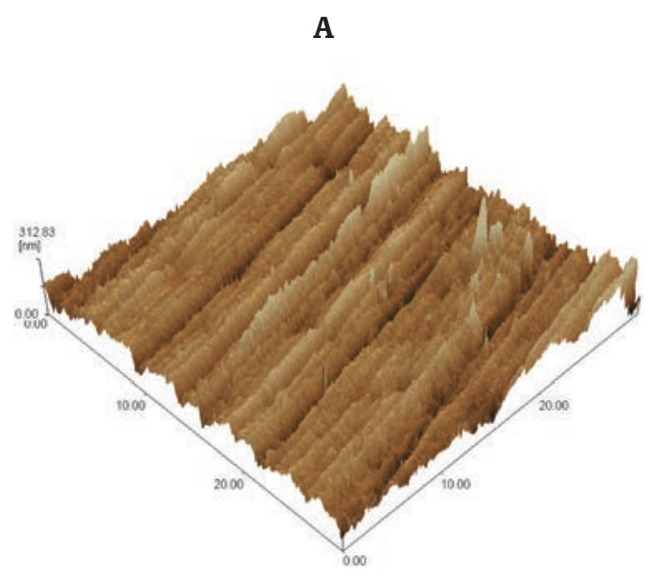

$30.00 \times 30.00[\mathrm{um}] \mathrm{Z} 0.00 \cdot 312.83[\mathrm{~nm}]$

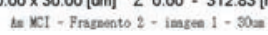

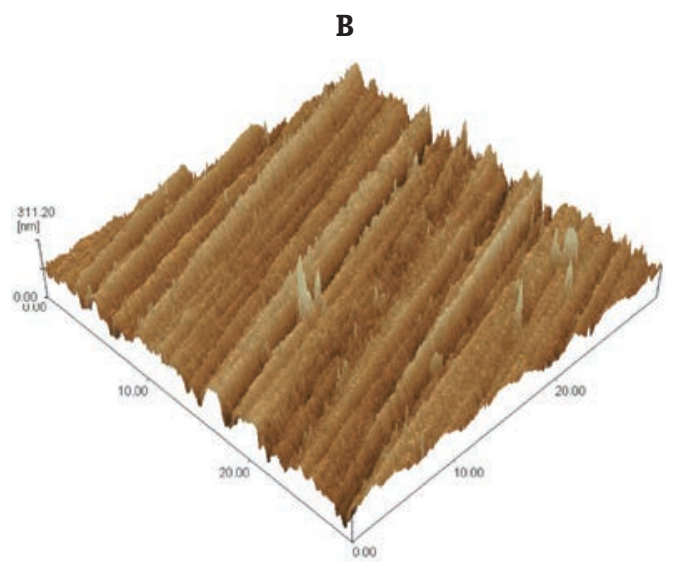

$30.00 \times 30.00[\mathrm{um}] \quad z 0.00-311.20[\mathrm{~nm}]$ An KCI - Fragento 2 - imagen 1 - 30 a

Figura 3. A: imagen MFA antes del procedimiento en el GC. B: imagen MFA después del procedimientos en el GC. 


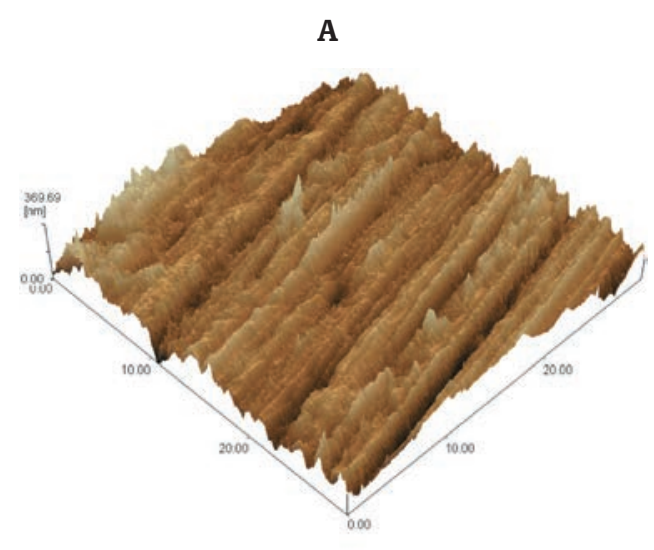

$30.00 \times 30.00[\mathrm{um}] \mathrm{Z} 0.00 \cdot 369.69 \mathrm{gmm}$ Aa XC1 - Fragento 1 - ingos $2-30 \mathrm{ue}$

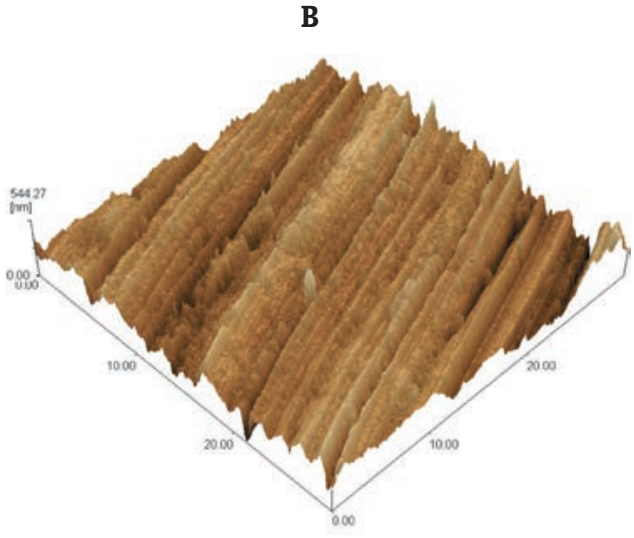

$30.00 \times 30.00[\mathrm{um}] \quad z \quad 0.00-544.27[\mathrm{~nm}]$

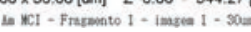

Figura 4. A: imagen MFA antes del procedimiento en el GCC. B: imagen MFA después del procedimientos en el GCC.

\section{DISCUSIÓN}

El cepillado dental es esencial para eliminación de la placa bacteriana y los residuos alimenticios a fin de contribuir con la buena salud dental. La American Dental Association (ADA) recomienda cepillarse dos veces al día con una aplicación de fuerza suave y de forma circular. ${ }^{20}$ Se ha determinado que la fuerza de aplicación en el momento de cepillado es de $250 \mathrm{~g}$ (2,5 a $3 \mathrm{~N}$ ) y con una duración de 120 segundos por día. ${ }^{18}$ Esto ha hecho que se desarrollen metodologías tanto en dentina como en esmalte para evaluar In vitro, los efectos de procesos de cepillado junto con precipitados de cremas dentales. ${ }^{21}$

Estudios como los de Ganss y colaboradores (2009), han determinado 120 segundos de cepillado por día, ${ }^{18}$ en este estudio se realizó un cepillado con crema dental con 1450 ppm de fluor y se llevó a cabo por diez minutos, para simular los cinco días después del proceso de aclaramiento y evaluar la influencia de este proceso junto con el precipitado, en el esmalte dental.

Existe una gran controversia entre los valores encontrados de dureza superficial posteriores al aclaramiento, se observan estudios que no encuentran alteraciones significativas sobre la estructura del esmalte con el uso de peróxido de hidrógeno a altas concentraciones, ${ }^{14-22}$ y otros que reportan efectos deletéreos significativos. ${ }^{23-24}$ En este estudio se planteó la posibilidad de remineralización del esmalte luego del aclaramiento dental con el proceso de cepillado, junto con un precipitado de crema dental obteniendo buenos resultados, sin contar con el proceso de remineralización salivar. Los valores de microdureza superficial fueron restablecidos y aumentados.
Los cambios en el contenido de mineral en la superficie del esmalte están directamente relacionados con los cambios en la microdureza. ${ }^{25}$ En el grupo que se realizó cepillado después del aclaramiento dental con peróxido de hidrogeno al 35\%, no se encontró disminución de la dureza superficial, lo que podría ser explicado por el uso de crema dental fluorada con 1450 ppm que crea un equilibrio entre la desmineralización y remineralizacion.26 Por otro lado, el ion flúor puede prevenir la disminución de la dureza superficial mediante la formación de una capa de calcio fluorado en la superficie del esmalte..$^{25-27}$

Las alteraciones del esmalte pueden darse también en la microformologia, aunque no son perceptibles clínicamente, ${ }^{28}$ estas pueden darse cuando se trata el esmalte con soluciones con un menor $\mathrm{pH}$, el cual puede disolverlo debido a la acidez. ${ }^{10,29}$ Los resultados obtenidos en rugosidad en el Grupo GC después de aclaramiento, coinciden con los obtenidos por Domínguez y colaboradores quienes no encontrando diferencias significativas luego de realizar cepillado por diez minutos. ${ }^{14}$

A partir de este estudio, se podría concluir:

El cepillado con crema dental con 1450 ppm de flúor, después del proceso de aclaramiento dental con peróxido de hidrógeno al 35\% mantiene la estabilidad de la dureza superficial del esmalte.

El cepillado con crema dental con 1450 ppm de flúor, después del proceso de aclaramiento dental con peróxido de hidrogeno al 35\% mantiene intacta la morfología superficial del esmalte.

\section{Agradecimientos}

Los autores desean agradecer al Técnico del Laboratorio de la U. Estadual de Ponta Grossa, Nilson Sa- 
bino por la colaboración en las imágenes de AFM, a la Universidad Santiago de Cali y a la Universidad Estadual de Ponta Grossa por permitir la realización en conjunto de este trabajo.

\section{BIBLIOGRAFÍA}

1. Christensen GJ. The state of the art in esthetic restorative dentistry. J Am Dent Assoc 1997; 128: 1315 - 1317.

2. Tin-Oo MM, Saddki N, Hassan N. Factors influencing patient satisfaction with dental appearance and treatments they desire to improve aesthetics. BMC Oral Health 2011; 23: 11 - 16.

3. Gomes MN, Francci C, Medeiros IS, De Godoy Froes Salgado NR, Riehl H, et al. Effect of light irradiation on tooth whitening: Enamel microhardness and color change. J Esthet Restor Dent 2009; 21: 387 - 394.

4. Toteda M, Philpotts CJ, Cox TF, Joiner A. Evaluation of a $6 \%$ hydrogen peroxide tooth-whitening gel on enamel microhardness after extended use. Quintessence Int 2008; 39: 853 - 858.

5. Attin T, Schmidlin PR, Wegehaupt F, Wiegand A. Influence of study design on the impact of bleaching agents on dental enamel microhardness: a review. Dent Mater. 2009; 25: 143 - 157.

6. Ernst $\mathrm{CP}$, Marroquin BB, Willershausen-Zönnchen $\mathrm{B}$. Effects of hydrogen peroxide-containing bleaching agents on the morphology of human enamel. Quintessence Int 1996; 27: 53 - 56.

7. Gultz J, Kaim J, Scherer W, Gupta H. Two in-office bleaching systems: A scanning electron microscopy study. Compend Contin Educ Dent 1999; 20: 965 - 970.

8. Sulieman M, Addy M, Macdonald E, Rees JS. A safety study in vitro for the effects of an in-office bleaching system on the integrity of enamel and dentine. J Dent 2004; 32 : $581-590$.

9. Tong LS, Pang MK, Mok NY, King NM, Wei SH. The effects of etching, micro-abrasion, and bleaching on surface enamel. J Dent Res 1993; 72: 67 - 71.

10. Hegedüs C, Bistey T, Flóra-Nagy E, Keszthelyi G, Jenei A. An atomic force microscopy study on the effect of bleaching agents on enamel surface. J Dent 1999; 27: 509 515.

11. Spalding M, Taveira LA, de Assis GF. Scanning electron microscopy study of dental enamel surface exposed to $35 \%$ hydrogen peroxide: Alone, with saliva, and with $10 \%$ carbamide peroxide. J Esthet Rest Dent 2003; 15: $154-165$.

12. Bitter NC. A scanning electron microscopy study of the effect of bleaching agents on enamel: A preliminary report. J Prost Dent 1992; 67: 852 - 855.

13. Pinto CF, Oliveira R, Cavalli V, Giannini M. Peroxide bleaching agent effects on enamel surface microhardness, roughness and morphology. Braz Oral Res 2004; 18: 306311.

14. Dominguez JA, Bittencourt B, Michel M, Sabino N, Gomes JC, Gomes OM. Ultrastructural evaluation of enamel after dental bleaching associated with fluoride. Microsc Res Tech 2012; 75: 1093 - 1098.

15. Worschech CC, Rodrigues JA, Martins LR, Ambrosano GM. Brushing effect of abrasive dentifrices during at-home bleaching with $10 \%$ carbamide peroxide on enamel surface roughness. J Contemp Dent Pract 2006; 7: 25 - 34.
16. Hefferren JJ. A laboratory method for assessment of dentifrice abrasivity. J Dent Res 1976; 55: 563 - 573.

17. Franzò D, Philpotts CJ, Cox TF, Joiner A. The effect of toothpaste concentration on enamel and dentine wear In vitro. J Dent 2010; 38: 974 - 979.

18. Ganss C, Schlueter N, Preiss S, Klimek J. Tooth brushing habits in uninstructed adults--frequency, technique, duration and force. Clin Oral Investig 2009; 13: 203 - 208.

19. Borges $A B$, Yui KC, D'Avila TC, Takahashi CL, Torres $C R$, Borges AL. Influence of remineralizing gels on bleached enamel microhardness in different time intervals. Oper Dent 2010; 35: 180-186.

20. American Dental Association. Oral Health Topics A-Z. Cleaning Your Teeth and Gums (Oral Hygiene). URL disponible en: http://www.ada.org/public/topics/cleaning.asp

21. Prati C, Venturi L, Valdrè G, Mongiorgi R. Dentin morphology and permeability after brushing with different toothpastes in the presence and absence of smear layer. J Periodontol 2002; 73: 183 - 190.

22. Sulieman M, Addy M, Macdonald E, Rees JS. A safety study in vitro for the effects of an in-office bleaching system on the integrity of enamel and dentine. J Dent 2004; 32: $581-590$.

23. Lewinstein I, Fuhrer N, Churaru N, Cardash H. Effect of different peroxide bleaching regimens and subsequent fluoridation on the hardness of human enamel and dentin. J Prosthet Dent 2004; 92: 337 - 542.

24. Al-Salehi SK, Wood DJ, Hatton PV. The effect of $24 \mathrm{~h}$ nonstop hydrogen peroxide concentration on bovine enamel and dentine mineral content and microhardness. J Dent 2007; 35: 845 - 550.

25. Attin T, Kielbassa AM, Schwanenberg M, Hellwig E. Effect of fluoride treatment on remineralization of bleached enamel. J Oral Rehabil 1997; 24: 282 - 286.

26. Bizhang M, Seemann R, Duve G, Römhild G, Altenburger JM, Jahn KR, Zimmer S. Demineralization effects of 2 bleaching procedures on enamel surfaces with and without post-treatment fluoride application. Oper Dent 2006; 31: 705 - 709.

27. Cate JM, Arends J. Remineralization of artificial enamel lesions in vitro. Caries Res 1977; 11: 277 - 286.

28. Markovic L, Jordan RA, Lakota N, Gaengler P. Micromorphology of enamel surface after vital tooth bleaching. J Endod 2007; 33: 607 - 610.

29. Driessens FC, Theuns HM, Borggreven JM, van Dijk JW. Solubility behaviour of whole human enamel. Caries Res 1986; 20: 103 - 110.

\section{Correos electrónicos de los autores:}

John Alexis Domínguez: johnalexis.dominguez@gmail.com María Alejandra Paredes Marín: majanparedes@gmail.com Karol Andrea Gómez Ballesteros: shiremn9@gmail.com Gustavo Adolfo Martínez Alvarado: cheo0619@gmail.com Gustavo Hannover Alegría Acevedo: alegriagus@hotmail.com Osnara María Mongruel Gomes: osnaramgomes@uol.com.br 